\section{Solvent Microextraction: Theory and Practice}

by John M. Kokosa, Andrzej Przyjazny and Michael A. Jeannot: Wiley, Hoboken, NJ, USA, 2009, pp ix+323, ISBN: 978-0-470-27859-8.

Price: EUR 99.99, USD 115.00

The efforts made in the sample preparation field in the past 15 years have led to the adaptation of existing methods and the development of new techniques in order to save time and chemicals, improve overall performance and, if possible, hyphenate the various steps of the analytical process. In this context, miniaturization has become a key factor and frequently the best alternative, especially when dealing with small size samples.

This book reviews the several solventbased microextraction techniques developed since the introduction of the concept by discussing their respective advantages and limitations, the successive modifications and applications in the field, as well as the current state-of-the-art and the latest trends in this active research area. The text is organised in seven chapters. In the first chapter, the basic concepts and main features of solvent microextraction (SME) are compared with those of nowadays well established solvent- and sorbent-based sample preparation techniques. Chapter 2 provides a more detailed discussion on the several SME operation modes and describes the general methodology and basic instrumentation required for the various configurations and possible approaches. The physical and chemical principles involved in the SME process are discussed in Chap. 3, where the different variables affecting the extraction process and the kinetics of the SME techniques are evaluated. Practical considerations regarding the different SME modes are presented in Chap. 4. Interestingly, the theoretical aspects discussed in the previous chapter are demonstrated here through basic calculations applied to the most common SME modes. A practical and illustrative approach to deciding the most appropriated SME format for each particular application is suggested in Chap. 5, which also includes a detailed discussion of the factors to consider in the several steps of SME development for liquid samples. Chapter 6 is an exhaustive review of the literature published on SME since the introduction of the technique up to December 2008, organised by test matrix, application field and measurement of physicochemical properties. Some selected applications are further discussed offering a detailed "how to" in Chap. 7 following a common format including description of the case study, experimental conditions, results and discussion and additional experimental recommendations based on the experience of the authors. The final appendix summarises an interesting glossary of terms in a valuable attempt to unify the diverse nomenclature still used for these techniques. The book includes a CD containing more the 600 references and some illustrative tables describing selected applications of SME, which represents a good starting point for readers who want to obtain a general overview of the current state-of-the-art for these miniaturised techniques.

Written by three pioneers and worldwide recognised specialists in the field, this timely monograph is not only an exhaustive and updated compilation of bibliography related to solvent microextration techniques and their applications in different work areas, but a practical and useful guide for all those interested in their direct application to practical case studies.

L. Ramos 